\title{
Measuring the supermassive black hole parameters with space missions
}

\author{
Alexander F. Zakharov \\ National Astronomical Observatories of Chinese Academy of Sciences, Beijing 100012, China \\ ITEP, 25, B. Cheremushkinskaya st., Moscow 117259, Russia \\ BLTP, JINR, Dubna, Russia \\ email: zakharov@itep.ru
}

\begin{abstract}
Recent X-ray observations of microquasars and Seyfert galaxies reveal broad emission lines in their spectra, which can arise in the innermost parts of accretion disks. Recently Müller \& Camenzind (2004) classified different types of spectral line shapes and described their origin. Zakharov (2006b) clarified their conclusions about an origin of doubled peaked and double horned line shapes in the framework of a radiating annulus model and discussed s possibility to evaluate black hole parameters analyzing spectral line shapes.
\end{abstract}

Keywords. Black holes - X-ray astronomy - supermassive black holes

There are a lot of papers discussing theoretical aspects of possible scenarios for generation of broad iron lines in AGNs, see for example, reviews by Fabian et al. (2000); Matt (2006). Moreover, an influence of microlensing on Fe $K_{\alpha}$ line shapes and spectra was discussed by Popovic et al. (2006); optical depths for these phenomena were calculated by Zakharov et al. (2004, 2005a,b). Formation of shadows (mirages) is another example when general relativistic effects are extremely important and in principle they could be detected with forthcoming interferometrical facilities such as Radioastron, Millimetron, MAXIM, as was shown by Zakharov et al. (2005c,d,e,f,g,h) (perspective studies of microlensing with Radioastron facilities were discussed (Zakharov (2006a)). Observations of shadows could give a real chance to observe "faces" of black holes of black holes and confirm general relativity predictions in the strong gravitational field, and to obtain new constraints on alternative theories of gravity.

Müller \& Camenzind (2004) classified different types of spectral line shapes and described their origin. Zakharov (2006b) and Zakharov \& Repin (2005, 2006) clarified their conclusions about an origin of doubled peaked and double horned line shapes. Based on results of numerical simulations we showed using a radiating annulus model that double peaked spectral lines arise for almost any locations of narrow emission rings (annuli) (except closest orbits as we could see below) although Müller \& Camenzind (2004) concluded that such profiles arise for relatively flat spacetimes and typical radii for emission region about $25 r_{g}$. We did not impose assumptions about an emissivity law; we only assume that the radiating region is a narrow circular ring (annulus). We used an approach which was discussed in details by Zakharov (1991a); Zakharov (1994a); Zakharov (1995, 2003, 2004, 2005); Zakharov \& Repin (1999, 2002, 2003a,b,c, 2004a); Zakharov et al. (2003, 2004). The model is based on results of qualitative analysis done earlier by Zakharov (1986, 1988, 1989, 1991b).

\section{Acknowledgements}

AFZ is grateful to the National Natural Science Foundation of China (NNSFC) (Grant 10233050) and National Key Basic Research Foundation of China (Grant TG 2000078404) for a partial financial support of the work. 


\section{References}

Fabian, A. C., Iwazawa, K., Reynolds, C. S. \& Young, A. J. 2000, PASP, 112, 1145

Matt, G. 2006, Astronomische Nachrichten, 327, 949

Müller, A. \& Camenzind, M. 2004, A\&A, 413, 861

Popović, L. C., Jovanović, P., Mediavilla, E., Zakharov, A. F., Abajas, C. et al. 2006, AJ, 637, 630

Zakharov, A. F. 1986, Sov. Phys. - J. Exper. Theor. Phys., 64, 1

Zakharov, A. F. 1988, Soviet Astron., 32, 456

Zakharov, A. F. 1989, Sov. Phys. - J. Exper. Theor. Phys. 68, 217

Zakharov, A. F. 1991a, Soviet Astron., 35, 30

Zakharov, A. F. 1991b, Soviet Astron., 35, 147

Zakharov, A. F. 1994a, MNRAS, 269, 283

Zakharov, A. F. 1994b, Classical \& Quantum Gravity,, 11, 1027

Zakharov, A. F. 1995, in H. Böhringer, G. E. Morfill, J. E. Trümper (eds.), Proc. of the 17th Texas Symposium on Relativistic Astrophysics, (Ann. NY Academy of Sciences), p. 550

Zakharov, A. F. 2003, Publ. of the Astron. Observatory of Belgrade, 76, 147

Zakharov, A. F. 2004, in: L. Hadzievski, T. Gvozdanov, and N. Bibić (eds.) The Physics of Ionized Gases (AIP Conf. Proc.), Vol. 740, p. 398

Zakharov, A. F. 2005, Intern. J. Mod. Phys. A, 20, 2321

Zakharov, A. F. 2006a, Astronomy Reports, 50, 79

Zakharov, A. F. 2006b, Physics of Atomic Nuclei, 37, 1

Zakharov, A. F., Kardashev, N. S., Lukash, V. N. \& Repin, S. V. 2003, MNRAS, 342, 1325

Zakharov, A. F., Ma, Z. \& Bao, Y. 2004, New Astronomy, 9, 663

Zakharov, A. F., Nucita, A. A. \& De Paolis, F., Ingrosso G. 2005c, New Astronomy, 10, 479

Zakharov, A. F., Nucita, A. A., De Paolis, F. \& Ingrosso, G. 2005d, in: G. Vilasi, G. Espositio, G. Lambiase, G. Marmo, G. Scarpetta (eds.), Proc. of the 16th SIGRAV Conference on General Relativity (AIP Conf. Proceedings), Vol. 751, p. 227

Zakharov, A. F., Nucita, A. A., De Paolis, F. \& Ingrosso, G. 2005e, in: P. Chen, E. Bloom, G. Madejski, V. Petrosian (eds.), Proc. of the 22nd Texas Symposium on Relativistic Astrophysics (Stanford University: Stanford), PSN 1226

Zakharov, A. F., Nucita, A. A., De Paolis, F. \& Ingrosso, G. 2005f, in: H. V. KlapdorKleingrothaus and D. Arnowitt (eds.), Proc. of "Dark Matter in Astro- and Particle Physics", (Springer: Heidelberg), p. 77

Zakharov, A. F., Nucita, A. A., De Paolis, F. \& Ingrosso, G. 2005j, A\&A, 442, 795

Zakharov, A. F., Nucita, A. A., De Paolis, F. \& Ingrosso, G. 2005h, in: J. Tran Thanh Van and J. Dumarchez (eds.) Proc. of XXXXth Rencontres de Moriond "Very High Energy Phenomena in the Universe", (The GIOI Publishers), p. 223

Zakharov, A. F. Popović, L. C. \& Jovanović, P. 2004, A\&A, 420, 881

Zakharov, A. F., Popović, L. C. \& Jovanović, P. 2005a, in: Y. Mellier \& G. Meylan (eds.), Proc. of IAU Symposium, "Gravitational Lensing Impact on Cosmology", (Cambridge University Press: Cambridge), vol. 225, p. 363

Zakharov, A. F., Popović, L. C. \& Jovanović, P. 2005b, in: Y. Giraud-Heraud and J. Tran Thanh Van and J. Dumarchez (eds.) Proc. of XXXIXth Rencontres de Moriond "Exproring The Universe", (The GIOI Publishers), p. 41

Zakharov, A. F. \& Repin, S. V. 1999, Astronomy Reports, 43, 705

Zakharov, A. F. \& Repin, S. V. 2002, Astronomy Reports, 46, 360

Zakharov, A. F. \& Repin, S. V. 2003a, A\&A, 406, 7

Zakharov, A. F. \& Repin, S. V. 2003b, Astronomy Reports, 47, 733

Zakharov, A. F. \& Repin, S. V. 2003c, Nuovo Cimento, 118B, 1193

Zakharov, A. F. \& Repin, S. V. 2004, Adv. Space Res., 34, 1837

Zakharov, A. F. \& Repin, S. V. 2005, Mem. S. A. It. Suppl., 7, 60

Zakharov, A. F. \& Repin, S. V. 2006 New Astronomy, 11, 405 